\title{
Strategy and skills for moral decision-making in business
}

\author{
G.J. Rossouw \\ Philosophy Department \\ Rand Afrikaans University \\ JOHANNESBURG
}

\begin{abstract}
Strategy and skills for moral decision-making in business

Business Ethics is a truly interdisciplinary field of study. The specific issue of moral decision-making within the field of business ethics testifies to this. Recently some have made important contributions in this regard contributions in which they emphasised that moral theory is not sufficient for moral decision-making. What is needed besides moral theory is problem-solving ability. In this article the same point is argued, but from a philosophical perspective. It is further indicated that problem-solving ability entails more than merely a strategy for making moral decisions. It should also include the developmem of the thinking skills which are demanded by the strategy chosen for problem resolution.
\end{abstract}

\section{Introduction}

That business ethics is a truly interdisciplinary field of study, is evident from an analysis of the contributors to the academic journals on Business and Professional Ethics. It is equally evident that contributors from various disciplines tend to approach this interdisciplinary field of study differently. Jones (1982:211) for example, made a broad typology of the differences between the concerns of the various disciplines which contribute to the field of business ethics. He contended that theologians seems to be more interested in macro-economic ethical issues about the moral legitimacy of economic systems. The academic discipline of business management seems to be more focused on the meso-economic issues about the interaction between business and society. Philosophers, according to this typology, tend to engage themselves more with issues on the micro-economic level about the morality of specific business actions. Participation in the field of business ethics has fortunately not been restricted to these three role players. Recently contributions by psychologists have become more prominent. The issues about moral decision-making raised by psychologists through their contributions within the field of business and professional ethics will be explored from a philosophical perspective in this article. 


\section{Business ethics and moral decision-making}

When philosophers approach the field of business ethics, their contributions tend to be in the form of applied ethics. In this form it applies moral theory to practical issues in business. A glimpse at textbooks on business ethics written by philosophers testifies to this fact (cf. Olen \& Barry 1996; Velasquez 1992; Beauchamp \& Bowie 1993; Boatright 1993; White 1993). In most cases one finds that textbooks start by explaining the content of classical moral theories. Thereafter these theories are applied - often by means of a series of contemporary readings - to topical issues in contemporary business. The assumption behind this approach clearly is that moral theories can assist one in making decisions about difficult moral issues in business. What most textbooks, however, fail to do, is to show how one should move from competing - and often irreconcilable - moral theories to moral solutions. What is thus evidently lacking is the process of decision-making that should bridge the gap between moral theory and practical moral solutions

Recently this flaw in the philosophical approach to business ethics has been exposed by psychologists working in the field of business or professional ethics (cf. Gawthrop \& Uhlemann 1992; Kavathatzopoulos 1993; Kavathatzopoulos 1994). They have argued that moral theories on their own are inadequate for making moral decisions. What is needed for moral decision-making is a specific strategy that can produce practical solutions to moral problems. They have further demonstrated that training in moral decision-making strategy is effective to secure a transition from heteronomous to autonomous moral decision-making. In this respect they have enriched the study of business ethics by securing greater attention for the process of moral decision-making. Their contribution should thus be regarded as a valuable contribution to the ongoing discourse about business ethics.

Contributions to discourses always tend to evoke further contributions. This article is intended to be a response from a philosophical angle to this specific contribution that was made. The response will have two dimensions. On the one hand the suspicion that the mentioned psychologists seem to harbour agannst the value of moral theories for moral decision-making will be supported from a philosophical perspective. On the other hand, an aspect of moral decisionmaking that seems to be neglected in the mentioned contributions will be attended to. That aspect is the thinking skills needed for applying any moral decisionmaking strategy. 
G.J. Rossouw

\section{Moral theory and moral decisions}

It has already been stated that philosophical textbooks on business ethics seem to suggest that moral theories are a good point of departure for making moral decisions. This assumption, however, needs further examination.

Ethical theories are theories about the justification of moral actions. They propose appropriate reasons on which moral decisions should be based. Traditionally two major kinds of moral theories are distinguished, viz. deontological and consequensialist theories. Recently more theories were added, such as virtue theories and narrative theories. When ethical theories are compared, it is evident that there is no consensus on what these alleged appropriate reasons are that should ground our moral decisions (MacIntyre, 1981:5).

Kant, as the classical representative of deontological theories, contends that we should dutifully obey the imperatives for moral behaviour that is evident to all rational persons. Mill, representing a specific strand of consequentialism, argues that the amount of collective happiness rendered by actions should be the basis for our moral decisions. Narrative theories, again assert that the symbolic universe in which our lives are situated should determine what course of action we should follow in our moral decision-making. And so we can continue listing the alleged appropriate reasons which should gird our moral decisions.

Comparing just the three mentioned theories, it is evident that each of them opted for a different criterium for morality. Though none of these criteria are uncontroversial, each of them can be rationally justified. In that sense none of them can be dismissed as nonsensical, but at the same time none of them can claim superiority. Far from pointing out the one and only way toward moral decisionmaking, they rather formulate the various reasons that were historically advanced to ground moral decisions. There is no neutral referee available to decide which moral theory is best. It is exactly for this reason that the contemporary culture is described as a culture of moral dissensus.

This phenomenon of moral dissensus does not imply that moral theories should be dismissed as meaningless and in a state of mess. Moral theories still have value. They can help moral agents understand themselves better, in the sense that they provide detailed rational explanations for convictions that many people are only able to express as gut-feelings. Knowledge of moral theories can also sensitise one for the strengths and weaknesses of particular moral theories. They can assist one in becoming more tolerant towards persons who differ from you, because one will realise that within our culture of moral dissensus the moral theory that one subscribes to does not enjoy a monopoly status 
Although moral theories are useful for moral awareness and for understanding moral discourse, their usefulness in real decision-making about moral issues in business is restricted by various factors, of which two will be mentioned here:

- Moral dissensus is not only typical of post-modern culture in general, but also of business and more specifically large corporations. This moral dissensus has important implications for moral decision-making on disputes in which more than one person or party is involved. If we assume for the moment that people do indeed use moral theories as the basis for their moral decisions, then the question inevitably arises what should be done when persons who adhere to different moral theories propose conflicting solutions for the dispute. Moral theories themselves cannot lead us out of this impasse, because it has already been stated that it is impossible to judge one moral theory superior to another, because they are all in principle rationally and morally valid. Something more than moral theory is needed to solve this dilemma. What is needed is a strategy for moral decision-making and the thinking skills required by that strategy. This will be discussed later in this article.

- A further restriction of moral theories has to do with their nature. A moral theory proposes an abstract and clearly defined rule or principle that should guide all our actions. The situations that require moral judgement do not display the same features. On the contrary, situations in which we need to make moral decisions are concrete, clouded and controversial. They simply do not permit moral theories to be applied easily and neatly (cf. Vermaak, 1995:160). Solving moral dilemmas often demands difficult compromises and a creative mixture of various moral considerations. Moral theories once again might prove to be most helpful in understanding and analysing moral dilemmas, but they cannot produce solutions to moral problems. More than just moral theories are needed to produce practical solutions to moral problems.

It is to that "more" that the rest of this article will attend.

\section{Strategy for moral decision-making}

It has been argued till now that more than moral theory is needed for making moral decisions when faced with moral dilemmas. Psychologists indicated that the "more" that is needed is "problem solving ability" (cf. Kavathatzopoulos 1994:379). What will be explored next, is what "problem solving ability" entails as seen from a philosophical perspective. Such a perspective can enrich the mentioned psychological contributions to business ethics that seem to be merely concerned with facilitating a shift from what Piaget (1932) termed a heteronomous to an autonomous method of problem solving. What seems to be neglected is the quality of the decision-making process and the skills needed for 
making high quality decisions. Philosophy, through its tradition of inquiry first in logic, later in informal logic and most recently in the critical thinking movement (cf. Van Veuren, 1995) can offer perspectives that can contribute towards our understanding of and competency in "problem solving ability".

Problem solving in any domain always involves two things: strategy and execution. Strategy can be described as the plan according to which the problem at hand will be solved. Execution again, refers to performing the various steps or cognitive operations that the strategy demands for reaching a solution. In this section the focus will be on strategy and in the next section on the execution of the strategy.

A strategy for moral problem solving should of course take the limitations of moral theories discussed above, as well as the fact of moral dissensus, into consideration. What is thus needed is a strategy for moral decision-making that does not rely too heavily on moral theories and that also give due credit to the fact of moral dissensus.

A proposal for a strategy that meets these demands is the RIMS strategy for group decision-making that was previously published in Koers (cf. Rossouw, 1993:283-298; also see Rossouw 1994:53-71). RIMS is an acronym for Rational Interaction for Moral Sensitivity. The rationale behind this strategy will not be explained fully here, because it has already been done at length in the publications just referred to. The basic assumptions underlying the RIMS strategy will merely be mentioned briefly. They are the following

- Firstly, it assumes that moral dissensus is an inescapable feature of our current culture. Modernity, in its attempt to find a secular and rational ground for morality, produced various secular and rational grounds for morality. All of these grounds are rationally justifiable and defensible. This resulted in the current situation of dissensus, where none of the competing moral theories can succeed in gaining superiority over the others. They therefore need to be taken either equally seriously, or all of them need to be rejected. The first option forms the first assumption of the RIMS strategy.

- Secondly, it assumes that moral dissensus does not neccesarily result in ethical relativism. Moral dissensus only equals ethical relativism, if it is assumed that discourse between the rival moral viewpoints has become meaningless. This is not the case in the RIMS strategy. It rests on the assumption that interaction between rival moral viewpoints is not only neccesary, but is also an important source of creativity that can assist all involved in finding moral decisions that are more morally sensitive. 
- Thirdly, it assumes that through dialogue conflicting moral views can be creatively harnessed to produce morally sensitive solutions to moral dilemmas. The preconditions for such a dialogue are that the reality of moral dissensus is understood and accepted by all participants in the RIMS strategy, and that participants commit themselves to finding a solution amidst the moral dissensus. Once this has occurred, the rivalry between moral viewpoints no longer frustrates moral decion-making, but becomes a creative resource that offers the opportunity of finding more comprehensive and morally sensitive solutions to the problem under discussion than any of the rival moral viewpoints can achieve on its own.

- Fourthly, it assumes that a focus on the motives underlying moral viewpoints cannot solve moral dilemmas in a situation of moral dissensus. The argument by Alisdair MacIntyre (1981:3-20) that rival moral views can be relayed to rival subjective convictions on what constitutes the good, is taken as a valid. Once this is granted and moral dissensus is accepted as a reality in current culture, it follows that a mere focus on underlying motives cannot solve moral problems amidst moral dissensus. At most a focus on moral motives can illuminate the various moral view points, but it cannot overcome the rivalry between conflicting moral viewpoints. For this reason the RIMS strategy advises that motivations underlying moral views should not become the main focus point of the dialogue between participants. The focus should rather be on finding solutions that can accommodate the concerns of all involved.

- Fifthly, it assumes that proper moral decisions can only be taken on the basis of balanced and reliable information. This information is provided by generating all the moral arguments which participants can identify on the issue under discussion. Before such arguments are accepted into the dialogue, participants should ensure that everyone involved in the dialogue understands the argument and that there is no factually false information included in the argument. This of course does not mean that all participants should agree with an argument before it can be included in the dialogue. It only implies that the arguments identified should be recognised as clear moral arguments.

This decision-making strategy can be broken down into three steps. They are:

\section{Step 1: Generate and audit arguments}

Any moral argument that satisfies the following three criteria should be taken into consideration in the decision-making process:

- The argument should not only take the interests of the person or party presenting it, into consideration, but should also consider the interests of other persons affected by the issue under discussion. 
- The argument should be clear and intelligible to other persons involved in the decision about the issue under discussion.

- The argument should be factually correct and logically coherent.

\section{Step 2: Identify consequences}

Persons involved in the decision-making process should avoid as far as possible focusing on one another's motives or moral presuppositions and should rather focus on the positive and negative implications identified by the various arguments.

\section{Step 3: Find solutions}

Participants should co-operate in finding solutions that will restrict these identified negative implications to a minimum, while retaining as far as possible the positive concerns identified in the previous step. (For an illustration of how the RIMS strategy can be applied to the moral disputes on affirmative action and compulsory AIDS testing respectively, see Rossouw, 1994:72-92 and 93-110.)

The mere application of a problem-solving strategy, such as the RIMS strategy, to a moral dispute does not guarantee that the said strategy will be properly executed. In order to apply a strategy such as the RIMS one properly, certain cognitive competence is needed - in other words, one needs the appropriate thinking skills that is implied by the strategy. It is exactly this element, viz. the teaching of thinking skills that is needed for applying a strategy for problem solving properly, that seems to be neglected in the specific psychological contributions on problem solving referred to at the beginning of this article.

In the rest of the article an indication will be given of the kind of thinking skills needed for proper problem solving. The RIMS strategy will again be used to illustrate what thinking skills are required by such a problem solving strategy. Other moral problem solving strategies will of course require other thinking skills, but my contention is that no strategy can be executed properly unless persons using those strategies have acquired the thinking skills required by the specific strategy they are using. Some remarks about the teaching of these skills will also be offered.

\section{Thinking skills for moral decision-making}

The thinking skills that are required to execute each of the three steps of the RIMS strategy properly are the following: 


\section{Step 1 :}

- Argumentation skills: In order to provide the information on which the ultimate decision will be based, the various arguments for and against the issue under dispute should be identified. This implies that one should know what an argument is and have the ability to formulate arguments. One should also be able to identify implied arguments as well as assumptions that have not been spelled out explicitly in some arguments.

- Identification of moral arguments: Moral decisions can only be based on moral arguments. It is therefore important that participants should also be able to distinguish moral arguments from amoral and immoral arguments. They should also be able to identify ego-centric and socio-centric arguments.

- Clarity and coherence: Information is only useful when it is clear and intelligible. This implies that arguments should be stated clearly. Key concepts in arguments should be well defined in order to eliminate vagueness and ambiguity. Arguments should also be formulated in a logically coherent way in order to make them clear and understandable to all involved.

- Intellectual tolerance: In order to give all arguments that meet the three criteria spelled out in step one of the RIMS strategy due consideration, participants also need intellectual tolerance. If they lack this virtue they will prematurely dismiss arguments that they do not agree with. (Strictly speaking intellectual tolerance is a trait of mind (virtue) rather than a thinking skill, but for the purposes of this paper a broad notion of thinking skills will be used that include virtues as well.)

\section{Step 2:}

- Distinguish motives from arguments: The RIMS strategy demands that motives should be avoided as far as possible. In order to avoid dwelling on motives, participants should of course be able to separate an argument from the motives underlying the argument. Participants should also be able to exercise meta-cognitive control in order to avoid straying into motives.

- Identify implications: The RIMS strategy further demands that the focus should be on the implications or concems identified through the various arguments. The skill that is needed here, is the ability to identify the consequences or concerns of each of the arguments presented in the dialogue. When the implication(s) of an argument is not spelled out explicitly participants should also have the ability to formulate implied implications. Furthermore participants should also have the ability to distinguish positive concerns from negative ones. 
Step 3

- Making compromises: Participants need to find a way out of the impasse created by conflicting viewpoints presented by moral arguments for and against the issue under discussion. In order to do that they need the ability to find positions that will minimise the negative consequences/concerns identified, while at the same time retaining the positive consequences/concerns that have been identified.

- Creative thinking skills: The fact that there is a moral dispute, suggests that existing approaches or proposals are not effective in solving the dispute. There is thus a need for finding new ways of dealing with the issue at hand This implies a need for creative thinking skills. Various techniques to stimulate innovation can be utilised in this regard, such as brainstorming, opposites, visualisation, and forced association to name but a few.

Each of these skills can and should be taught. If only decision-making strategy is taught, participants in the decision-making process will only learn how to make decisions and not how to make them properly. Teaching them the thinking skills that a specific strategy demands, enables them to make their moral decisions properly. This is not only true for the RIMS strategy but for all other decisionmaking strategies. Each strategy demands specific thinking skills in order to apply the strategy properly and thus to render a high quality result.

The teaching of thinking skills is a many faceted endeavour in which not only thinking skills should be addressed, but also matters such as the motivation to develop thinking skills, the virtues demanded for independent and innovative thinking, and factors that enable or disable one's ability to think properly (cf. Rossouw \& Lamprecht, 1995). It is impossible to cover these matters in this paper. Fortunately an abundance of literature available on these matters exists (like Paul, 1993, Collins \& Mangieri, 1992). Just as the field of business ethics offers opportunities for participation to many disciplines, so does the teaching of thinking skills for moral decision-making as well.

\section{Conclusion}

In reaction to contributions made by specific psychologists to the field of business ethics, and more specifically to moral decision-making in business ethics, two main arguments were raised in this article. Firstly, their claim that moral decision-making requires more than moral theories, was supported from a philosophical angle. Secondly, a certain dimension, essential to the execution of any moral decision-making strategy that seems to be neglected in the mentioned contributions, have been identified: the thinking skills which are required for the proper execution of a moral decision-making strategy. It was then illustrated 
Sirategy and skills for moral decision-making in business

what those thinking skills entail by using the RIMS strategy for moral decisionmaking as an example.

Although not an explicit objective of this article, it is also hoped that the article contibuted towards an understanding and appreciation of the importance and need of interdisciplinary work in the field of business ethics.

\section{Bibliography}

BEAUCHAMP, T.L. \& BOWIE, N.E 1993. Ethical theory and business Englewood Cliffs, NJ : Prentice Hall.

BOATRIGHT, J R. 1993. Ethics and the conduct of husiness. Englewood Cliffs, NJ Prentice Hall.

COLLINS, C. \& MANGIER, J.N. 1992. Teaching thinking. London : Lawrence Erlbaum Associates.

GAWTHROP, J.C. \& UHLEMANN, M R. 1992. Effects of problem-solving approach in ethics training. Professional Psychology: Research and Practice, 23(1):38-42.

JONES, D.G. 1982. Business, religion and ethics. Cambridge, MA : Oelgeschlager, Gunn and Hain.

KAVATHAZOPOULOS, I. 1993. Development of cognitive skill in solving business ethics problems: the effect of instruction. Journal of Business Ethics, 12: 379-386.

KAVATHAZOPOULOS, I. 1994. Training professional managers in decision-making about real life business ethics problems: the acquisition of the autonomous problem-solving skill. Journal of Business Ethics, 13:379-386.

MACINTYRE, A. 1981 A crisis in moral philosophy: why is the search for the foundations of ethics so frustrating? (In Callahan, D \& Engelhardt, H.T The roots of ethics. New York : Plenum Press p.)

OLEN, J. \& BARRY, V. 1996. Applying ethics. Belmont, CA : Wadsworth.

PAUL, R. 1993. Critical thinking. Sonoma, CA : Foundation for Critical Thinking

PIAGET, J. 1932. The moral judgment of the child London: Routledge \& Kegan Paul.

ROSSOUW, G.J. 1993. Moral decision-making amidst moral dissensus. Koers, 58(3):283298.

ROSSOUW, G.J. 1994 Business ethics: a Southern African perspective. Midrand Southern Books.

ROSSOUW, G.J. \& LAMPRECHT, J.C. 1995. Independent and innovative thinking Pretoria . Kagiso.

VAN VEUREN, P. 1995. Thinking skills in the context of Formal Logic, Informal Logic and Critical Thinking. Koers, 60(3):427-444

VELASQUES, M.G. 1992. Business ethics: concepts and cases. Englewood Cliffs, NJ : Prentice Hall

VERMAAK, M. 1995. Is a theory of argumentation necessary, desirable, possible? South African Journal of Philosophy, 14(4):159-161.

WHITE, T.I. 1993. Business ethics: a philosophical reader. New York : Macmillan 